\title{
VOCABULARY OF THE TANGOA LANGUAGE, ESPIRITU SANTO, NEW HEBRIDES.
}

\section{A L P H A BET.}

Vowels: a, e, i, o, u. Diphthongs: ai, au, oi, ao.

Consonants: b, c, g, $g, \mathrm{~h}, \mathrm{k}, \mathrm{l}, \mathrm{m}, m, \mathrm{n}, n, \mathrm{p}, p, \mathrm{r}, \mathrm{s}, \mathrm{t}, t, \mathrm{v}, \mathrm{w}, \mathrm{z}$.

The vowels and diphthongs are pronounced as in Italian. Of the consonants $\mathrm{c}$ is English $\mathrm{g}$ in go; $\mathrm{g}=$ English $\mathrm{ng}$ in sing; $g$ is a guttural trill, the melanesian $g ; m$ and $n$ are nasal; $p=$ pw or mpw; $t=t p$, a sound produced by the tongue between the teeth touching the upper lip; $\mathrm{v}$ closely approaches $\mathrm{b}$ and $\mathrm{w} . ; \mathrm{z}=$ ts.

$\mathrm{M}$ and $\mathrm{n}$ are often indifferently used, even by the same speaker.

\begin{tabular}{|c|c|c|c|c|c|}
\hline Abide & wonen & toko & beat & kloppen & vili \\
\hline act (n) & doen & sakasaka & because & omdat & natana \\
\hline afraid & bevreesd & nataku & beggar & bedelaar & tilabono \\
\hline again & weder & mele & belly & buik & page \\
\hline all & al & iseiso & bind & binden & lasi \\
\hline altar & altaar & vota & bird & vogel & nazi-abuabu ${ }^{3}$ \\
\hline animal & beest & nagi, magi & bite & bijten & kati \\
\hline ankle & enkel & valake & black & zwart & berika \\
\hline any & eenig & te & bleed & bloeden & rai \\
\hline arise & opstaan & turu & bless & zegenen & thatha-kina- \\
\hline arrow & pijl & evina. & & & ruku ${ }^{4}$ \\
\hline ascend & opklimmen & sake & blind & blind & buso \\
\hline ashes & asch & nābu-gabu 1 & blood & bloed & $\mathrm{rai}$ \\
\hline & & batu-mākovi ${ }^{2}$ & boat & boot & aka \\
\hline ask & v ragen & ausi & body & lichaam & sui \\
\hline & & & bone & been & sui \\
\hline $\mathrm{Bad}$ & slecht & sati & born, to be & geboren & bora \\
\hline banana & pisangboom & biatali & bosom & boezem & ruma \\
\hline basket & korf & taga & bow & & \\
\hline beard & baard & gumi & (weapon) & boog & baka \\
\hline
\end{tabular}




\begin{tabular}{|c|c|c|c|c|c|}
\hline bow down & buigen & pap uuki & day & dag & rani \\
\hline boy & jongen & natu-bariki 5 & death & dood & nateia 11 \\
\hline branch & tak & nasaga & deceive & bedriegen & kalu \\
\hline breast & borst & patu-susu & deep & diep & bua, buaka 12 \\
\hline bring & brengen & labi-nai 6 & descend & nederkomen & sibo, sebuti \\
\hline bring forth & $\begin{array}{l}\text { voortbren- } \\
\text { gen }\end{array}$ & labi-nai & $\begin{array}{l}\text { die } \\
\text { dirty }\end{array}$ & $\begin{array}{l}\text { sterven } \\
\text { vuil }\end{array}$ & $\begin{array}{l}\text { nate } \\
\text { lumim, berika }\end{array}$ \\
\hline oom & brem & tebes & divide & deelen & tope \\
\hline rother & broeder & tabai, tasi & do & doen & thai \\
\hline vild & bouwen & tiu & $\operatorname{dog}$ & hond & viriu \\
\hline urn & branden & suli & door & deur & talati, tarukua \\
\hline bury & begraven & tabuni & down & nederwaarts & ssibo, atano 13 \\
\hline butterfly & vlinder & $p$ е $p$ e & $\begin{array}{l}\text { dress }(n) \\
\text { drink }\end{array}$ & $\begin{array}{l}\text { kleeding } \\
\text { drinken }\end{array}$ & $\begin{array}{l}\text { ruru } \\
\text { enu, inu }\end{array}$ \\
\hline Call & roepen & tobi & dwell & wonen & toko \\
\hline carry & dragen & labi & & & \\
\hline chief & hoofd & moli, supe. & Ear & oor & pero \\
\hline child & kind & natu & earth & aarde & leta \\
\hline childless & kinderloos & maibu. & eat & eten & kani \\
\hline clean & zuiver & vuso & egg & el & sile \\
\hline clond & wolk & telagi & enemy & vijand & meresai. \\
\hline club & knuppel & maza & evening & avond & bogi, alo mo \\
\hline cocoanut & kokosboom & niu & & & sibo ${ }^{14}$ \\
\hline cold & koud & nakariri & every & ieder & tari \\
\hline come & komen & nai & expect. & verwachten & moloromromi \\
\hline $\operatorname{cool}(v)$ & koelen & nakariri & eye & oog & ata \\
\hline & dekken & tauri & & & \\
\hline covering $(n)$ & deksel & $\mathrm{kab}$ & Face & gezicht & ako \\
\hline crooked & krom & keeke & fall & vallen & ovi \\
\hline crumb & kruim & utautaki-kani 7 & far off & ver & $\mathrm{au}$ \\
\hline cry & weenen & tagi & $\begin{array}{l}\text { fatted } \\
\text { father }\end{array}$ & $\begin{array}{l}\text { vet } \\
\text { vader }\end{array}$ & $\begin{array}{l}\operatorname{taru} \\
\operatorname{tama}(\text { voc, tea) }\end{array}$ \\
\hline Dance & dansen & velu & fear & vrees & natku \\
\hline dark & duister & berika & feather & veder & vulu \\
\hline darken & verdonkeren & n bunbuni & feed & voeden & tha-kani ${ }^{15}$ \\
\hline darkness & duisternis & roroka & female & vrouwelijk & garai \\
\hline daughter & dochter & ve-natu ${ }^{8}$ & few & weinig & nakomo \\
\hline & & roro-rani & field & veld & zava, lobukai \\
\hline & da & $\left\{\begin{array}{c}\text { rani mo ka- } \\
\text { kara }^{10}\end{array}\right.$ & $\begin{array}{l}\text { find } \\
\text { finger. }\end{array}$ & $\begin{array}{l}\text { vinden } \\
\text { vinger }\end{array}$ & $\begin{array}{l}\text { bilel } \\
\text { tisu-lina } 16\end{array}$ \\
\hline
\end{tabular}




\begin{tabular}{|c|c|c|c|c|c|}
\hline fire & vuur & $g a b u$ & heavy & zwaar & bukoni \\
\hline first & eerst & teroma & here & hier & nigki, niki \\
\hline $\operatorname{sh}$ & visch & nazi-ki-tas ${ }^{17}$ & hereafter & hierna & pantako \\
\hline sh-hook & vischhaak & reberebe & hide & verbergen & bunbuni \\
\hline esh & vleesch & visiko & hill & heuvel & buti \\
\hline pwer & bloem & bira, bua & holy & heilig & $\mathrm{a}($ good $)$, ta- \\
\hline fly (insect) & vlieg & lago & & & \\
\hline fly $(v)$ & vliegen & rala bugo. & honour & eeren & ololo \\
\hline od & voedsel & kanikani, 18 & horns (?) & horen. & \\
\hline ot & voet & balo & house & huis & ima \\
\hline forefather & voorvader & pira & how? & hoe? & tamacia? \\
\hline fowl & hoen & toa & how many? & hoeveel & visa? \\
\hline friend & vriend & takasa & hungry & hongerig & tilabono \\
\hline ait & vrucht & bua & husband & $\operatorname{man}$ & tua \\
\hline el & $\begin{array}{l}\text { brandhout } \\
\text { vol }\end{array}$ & $\begin{array}{l}\text { gabu-nate } 20 \\
\text { naribi }\end{array}$ & hu & sch & \\
\hline & & & Image & beeld & tabu \\
\hline Garden & tuin & zara & inherit & erven & sipai \\
\hline gate & ingang & tarukua & inside & binnenzijde & nalolo \\
\hline gather & vergaderen & koko & island & eiland & uriâuri \\
\hline generation & geslacht & maka-pira & & & \\
\hline give & geven & sila & eep back & achterhou- & korokoro \\
\hline go & gaan & thano, mule & & 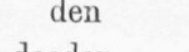 & \\
\hline go away & weggaan & thano-luke & kill & dooden & vili, tha-nate 22 \\
\hline go down & ondergaan & sebuti & kingdom & koninkrijk & tamata \\
\hline $\begin{array}{l}\text { go up } \\
\text { good }\end{array}$ & $\begin{array}{l}\text { opstijgen } \\
\text { goed }\end{array}$ & sak & & kussen & bugosi \\
\hline great & $\begin{array}{l}\text { goed } \\
\text { groot }\end{array}$ & tabera & & $\mathrm{m}$ & $\begin{array}{l}\text { sita } \\
\text { rogo-bosaki, } 23\end{array}$ \\
\hline ground & grond & leta & know & weten & $\begin{array}{l}\text { basaki, la-bo- } \\
\text { saki } 24\end{array}$ \\
\hline Tair & haar & vulu & & & \\
\hline allow & heiligen & tabu & Lad & jongen & natu-bariki \\
\hline land & hand & lima, lina & lame & lam & karizu \\
\hline ard & hard & rilagi & land & land & thanua \\
\hline hat & hoed & kabna-patu 21 & large & groot & tabera \\
\hline head & hoofd & patu & lead $(v)$ & voorgaan & tikau \\
\hline hear & honren & rog & leaf & blad & rat \\
\hline boont & hart & nabu & leap & springen & \\
\hline leaven & hemel & tuga, tuga-keza & lick & likken & \\
\hline
\end{tabular}


lie, falsehood lie down

lift

lift up eyes aanschouwen lukilau light (not

heavy) licht salesale lightning weerlicht baka like (arj.) gelijk soke like $(v$.$) \quad gaarne heb- boii$ ben

likeness gelijkheid niniu line hengelsnoer romai lip lip

vivi

live (life) leven live (dwell) wonen nauri

look

lose zien le toko ${ }^{25}$

louse

love

Make male

$\operatorname{man}$

inany

mast

mat

noon

nore

norning nosquito nother nountain nouth mond auch verliezen lukilau luis rausi beminnen boii$$
\text { nu }
$$

Vame

$$
\text { maken thai }
$$$$
\text { mannelijk lamani }
$$$$
\text { mensch tamloci, lamani }
$$
veel mast mat maan meer morgen muskiet moeder berg eck eighbour buur

\begin{tabular}{|c|c|c|}
\hline net & net & tara \\
\hline new & nieuw & baro \\
\hline night & nacht & bogi \\
\hline no & geen & ehe , supu \\
\hline one & geen & supu-tea 28 \\
\hline nose & neus & galisu \\
\hline not & niet & supu \\
\hline ld & oud & tuai, ${ }^{29}$ tatuai \\
\hline utside & buitenzijde & nazigo \\
\hline ver & boven & papatu, aulu 30 \\
\hline addle & pagaai & $\operatorname{ebose}(n)$ sua $(v)$ \\
\hline ersuade & overreden & rasu \\
\hline ig & varken & boi, ${ }^{31}$ pirura $^{32}$ \\
\hline it & kuil & moru \\
\hline ity & $\begin{array}{c}\text { medelijden } \\
\text { hebben }\end{array}$ & romi \\
\hline lace & plaats & zara \\
\hline lant & planten & $\operatorname{tani}(n)$ labo $(v)$ \\
\hline oison & vergift & biri \\
\hline porch & portaal & takura-ima 83 \\
\hline portion & deel & topea \\
\hline possess & bezitten & sipara \\
\hline praise & lof & reti-ruku ${ }^{34}$ \\
\hline unish & straffen & komo \\
\hline ut & stellen & tauu \\
\hline & nederleggen & \\
\hline put in & inzetten & soko \\
\hline Rain & regen & usa \\
\hline raise & opheffen & take \\
\hline 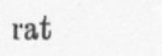 & rat & garibi \\
\hline r & rood & kakara \\
\hline ejoice & verheugen & abulaki \\
\hline main & wonen & toko \\
\hline emember & gedenken & leromromi \\
\hline pent & $\begin{array}{l}\text { berouwheb- } \\
\text { ben }\end{array}$ & leromromi \\
\hline & rust & nabu \\
\hline
\end{tabular}




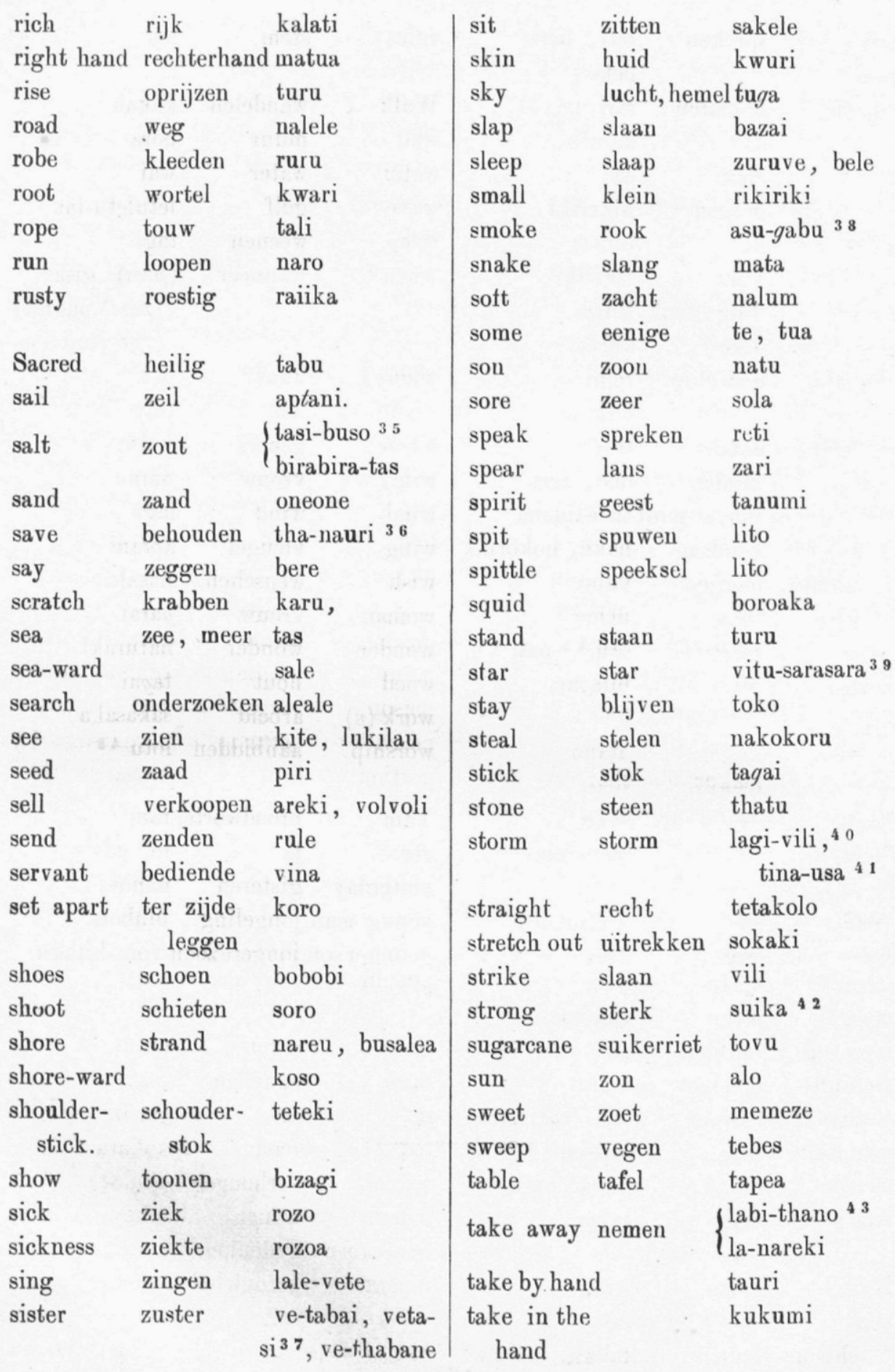




\begin{tabular}{|c|c|c|c|c|c|}
\hline $\begin{array}{l}\text { talk } \\
\text { taro }\end{array}$ & spreken & $\begin{array}{l}\text { reti, bere } \\
\text { peta }\end{array}$ & voice & stem & leo \\
\hline tear $(v)$ & scheuren & rari & Walk & wandelen & kakau \\
\hline hat & dat & karatu & wall & muur & bora \\
\hline 1ere & daar & ea & water & water & wai \\
\hline terefore & daarom & nikeriki & wave & golf & letuletu-tas \\
\hline ick & dik & tabera & weep & weenen & tagi \\
\hline in & dun & rikiriki & when? & wanneer & (pres) gisa? \\
\hline ling & ding, zaak & kinao & & & (past) nagisa? \\
\hline is & deze & kariki & & & (fut) pagisa?47 \\
\hline lought & gedachte & romi & where? & waar? & ei? \\
\hline row & werpen & bulaki & white & wit & Iulu \\
\hline iunder & donder & biri & whole & geheel & iseiso \\
\hline & binden & lico, reri & wife & vrouw & narau \\
\hline$p$ of finger & vinger-punt & tisu-linana & wind & wind & lagi \\
\hline day & vandaag & nake, nokoriki & wing & vleugel & aptani \\
\hline -morrow & morgen & vuho & wish & wenschen & nasalo \\
\hline ongue & tong & neme & woman & vrouw & garai \\
\hline oth & $\operatorname{tand}$ & oru, ${ }^{44}$ pati ${ }^{45}$ & wonder & wonder & naruraki \\
\hline & boom & bitagài & $\begin{array}{l}\text { wood } \\
\text { work }(n)\end{array}$ & $\begin{array}{l}\text { hout } \\
\text { arbeid }\end{array}$ & $\begin{array}{l}\text { tagai } \\
\text { sakasaka }\end{array}$ \\
\hline $\begin{array}{l}\text { Inder } \\
\text { nwilling }\end{array}$ & $\begin{array}{l}\text { onder } \\
\text { onwillig }\end{array}$ & $\begin{array}{l}\text { atano } \\
\text { roku }\end{array}$ & worship & aanbidden & lotu 48 \\
\hline & op & sake & Yam & broodwortel & ram \\
\hline$\mu$ & op & aulu $\operatorname{kin}^{46}$ & $\begin{array}{l}\text { yes } \\
\text { yesterday }\end{array}$ & $\begin{array}{l}\text { ja } \\
\text { gisteren }\end{array}$ & $\begin{array}{l}\text { io, po } \\
\text { nanovi }\end{array}$ \\
\hline ley & $\begin{array}{l}\text { dal } \\
\text { zeer }\end{array}$ & morumoru & $\begin{array}{l}\text { young man } \\
\text { younger sol }\end{array}$ & $\begin{array}{l}\text { jongeling } \\
\text { jongerezoon }\end{array}$ & $\begin{array}{l}\text { ulubou } \\
\text { vora-ki }\end{array}$ \\
\hline
\end{tabular}


NOTE ON THE LITERAL MEANING OF THE WORDS NUMBERED.

1 dust of fire

2 stone-burnt (?)

3 flying animal. Cf. fish

4. to make a thing good

5 riki $=$ little

6 take or carry hither

7 bits of food

8 female child. Cf. sister

9 break of daylight

10 daylight is red

11 ia forms verbal noun. Cf. die

$12 k a$ forms adjectives from nouns

13 on the ground

14 sun goes down

15 make eat

16 tip of hand

17 animal-of-sea. Cf. bird

18 animal food, flesh

19 vegetable food.

20 dead-fire

21 covering of head

22 make dead

23 hear and know

24 make to know.
25 continually dwell

26 place light, daylight

27 reduplication of bitti, hill

28 not one

29 formerly, of old.

30 on the head

31 a boar

32 a sow

33 gate of house

34 speak-good

35 sea-taste

36 make-live

37 ve feminine prefix. Cf. brother 38 smoke of fire

39 Cf. moon. sarasara $=$ every.

40 striking-wind. Cf. strike

411 mother of rain

42 bony

43 take hence. Cf. bring

4.4 front tooth

45 back tooth

46 on the head of

$47 n a=$ past tense, $p a=$ future

48 an introduced word 